\title{
Developing and testing theory- based and evidence-based interventions to promote switching to arsenic-safe wells in Bangladesh
}

Journal of Health Psychology 2014, Vol. 19(12) 1483-1498 (C) The Author(s) 2013 Reprints and permissions: sagepub.co.uk/journalsPermissions.nav DOI: $10.1177 / 13591053134938$ I। hpq.sagepub.com

@SAGE

\author{
Jennifer Inauen ${ }^{1,2}$ and Hans-Joachim Mosler'
}

\begin{abstract}
Millions of people in Bangladesh drink arsenic-contaminated water despite increased awareness of consequences to health. Theory-based and evidence-based interventions are likely to have greater impact on people switching to existing arsenic-safe wells than providing information alone. To test this assumption, we first developed interventions based on an empirical test of the Risk, Attitudes, Norms, Abilities and Self-regulation (RANAS) model of behaviour change. In the second part of this study, a cluster-randomised controlled trial revealed that in accordance with our hypotheses, information alone showed smaller increases in switching to arsenic-safe wells than information with reminders or information with reminders and implementation intentions.
\end{abstract}

\section{Keywords}

health behaviour, intervention, randomised controlled trial, social cognitions, theory

\section{Introduction}

Geogenic contamination of drinking water poses a severe threat to global health. In Bangladesh, 20 million people are at risk of drinking arsenic-contaminated water from shallow tube wells (Johnston and Sarker, 2007). The continuous consumption of arsenic can lead to arsenicosis, which comprises skin alterations, cardiovascular diseases and a variety of cancers (Smith and Steinmaus, 2009) and has been associated with increased mortality (Argos et al., 2010). As no cure for the disease has been found, preventing arsenicosis by drinking arsenic-safe water is the main mitigation approach. National and international agencies have laid great emphasis on mitigating the arsenic crisis by testing water from shallow tube wells and by raising awareness. However, many Bangladeshi people still use contaminated wells despite increased awareness of the threat of developing

\footnotetext{
'Eawag: Swiss Federal Institute of Aquatic Science \& Technology, Switzerland

2University of Konstanz, Germany

Corresponding author:

Jennifer Inauen, Department of Psychology,

Developmental and Health Psychology, University of Konstanz, P.O. Box 5560, 78457 Konstanz, Germany.

Email: jennifer.inauen@uni-konstanz.de
} 
arsenicosis (Johnston and Sarker, 2007), and despite the fact that many live within walking distance of a safe water alternative (Van Geen et al., 2002).

From a health psychology perspective, this finding is not astonishing. Evidence from studies on safe water consumption (Huber et al., 2012; Tobias and Berg, 2011) and other health behaviours has shown that risk perception is often a weak predictor of health behaviour change (e.g. Claassen et al., 2010; Radtke et al., 2011; Schwarzer and Luszczynska, 2008). Therefore, evidenced health behaviour change models take into account additional factors, for example, attitudes, social norms or self-regulation. Basing interventions on evidenced psychological theory should then produce a more successful behaviour change than conveying information alone (Michie et al., 2008; Michie and Johnston, 2012).

To determine factors that influence the use of safe drinking water options, we drew on the Health Action Process Approach (HAPA) (Schwarzer, 2008). This theory has successfully explained many health behaviours (e.g. Schwarzer, 2008). The HAPA includes the factors risk perception, outcome expectancies, self-efficacy and planning. To gain more intervention-relevant information, the HAPA factors were further disaggregated into several factors as depicted in the Risk, Attitudes, Norms, Abilities and Self-regulation (RANAS) model proposed by Mosler (2012) for water, sanitation and hygiene issues in developing countries.

In addition to the HAPA's risk perception, or perceived vulnerability, the RANAS also considers perceived severity and knowledge as behavioural determinants (Mosler, 2012). Perceived vulnerability is a person's subjective perception of his or her risk of contracting a particular condition or illness, and perceived severity is a person's perception concerning the seriousness of the consequences of contracting a particular condition or illness (Brewer et al., 2007).

Rather than considering the broad factor of outcome expectancies of the HAPA, the
RANAS separately takes into account attitude factors and norm factors (Mosler, 2012). Corresponding to the emotional and physical components of outcome expectancies of the HAPA (Schwarzer, 2008), research has shown that it is useful to distinguish between affective attitudes (e.g. enjoyable/not enjoyable) and instrumental attitudes (e.g. beneficial/ harmful) towards behaviour (Conner et al., 2011; Lawton et al., 2007).

For the social influences, several researchers have proved the usefulness of distinguishing between descriptive and injunctive norms (Conner and Sparks, 1996; Sheeran and Orbell, 1999). The descriptive norm expresses perceptions about which behaviours are typically performed. The injunctive norm reflects perceptions about which behaviours are typically approved or disapproved (Cialdini, 2003; Schultz et al., 2007). In direct correspondence to the HAPA, the RANAS also takes into account the three types of self-efficacy: action self-efficacy, maintenance self-efficacy and recovery self-efficacy (Schwarzer, 2008). Self-efficacy is described as the belief in one's capabilities to organise and execute the course of action required to manage prospective situations (Bandura, 1997).

In further correspondence with the HAPA (Schwarzer, 2008), the RANAS model specifies action planning and coping planning as volitional factors. Action planning is the specification of 'when', 'where' and 'how' the action shall take place, whereas coping planning is defined as the presumption of possible barriers and the invention of ways to overcoming them (Schwarzer, 2008). The RANAS also lists action control as an important factor of behaviour change (Mosler, 2012). It reflects efforts people carry out to perform intended behaviours (Sniehotta et al., 2005).

Two further self-regulation factors included in the RANAS, but not in the HAPA, are remembering and commitment strength (Mosler, 2012). Remembering refers to the ease of remembering to perform an action at a specific point in time or in a specific situation (Tobias, 2009). Several researchers have shown that the strength of the 
commitment to behaviour execution will modulate the effect of this event (e.g. Gollwitzer, 1999; Tobias, 2009).

Several publications have shown that factors in the RANAS model influence behaviour in the water and sanitation sector in developing countries. For solar water disinfection (SODIS), refer to the studies by Heri and Mosler (2008) in Bolivia and Kraemer and Mosler (2010) in Zimbabwe; for hygiene behaviour, see Graf et al. (2008) in Kenya; for consuming fluoridefree water from household or community filters, see Huber and Mosler (2012) and Huber et al. (2012); and for using arsenic-safe water options, refer to the studies by Inauen et al. (2013) and Mosler et al. (2010), both in Bangladesh.

Besides the compilation of most potential behaviour change predictors, a particular strength of the RANAS model is that it links specific factors to behaviour change techniques (BCTs). This is done in line with the joint efforts of several researchers to build a reliable taxonomy of BCTs (Abraham and Michie, 2008; Marks, 2009; Michie et al., 2013; Michie and Johnston, 2012). In addition to simply selecting the theoretically based factors, Mosler suggests an evidence-based approach, that is, to intervene on behavioural predictors that are most likely to promote behaviour change in the target population (Mosler, 2012). In brief, using intervention factors with the highest improvement potential is suggested, that is, factors with high predictive power as well as low mean values in the target population. In conclusion, the most effective interventions should be based on (a) behavioural determinants of sound psychological theory and (b) assessment of the factors with highest improvement potential with regard to the target population.

This procedure was adopted for the present study. In the first part, the behaviour change factors with the greatest improvement potential for promoting switching to arsenic-safe wells will be identified. Interventions will then be derived from these results to target the identified factors. In the second part of the study, these interventions will be combined with risk information and compared to an information-only control condition regarding their efficacy to promote behaviour change in a cluster-randomised controlled trial. Thereby, the assumption whether theory-based and evidence-based interventions increase the effects of informational interventions to promote the use of arsenic-safe water sources will be tested.

\section{Part I: Developing interventions by identifying psychological factors related to arsenic-safe water consumption}

In this part of the study, theory-based and evidence-based interventions to increase the use of arsenic-safe wells are developed by identifying the RANAS factors that are related to the use of arsenic-safe wells.

\section{Methods}

A cross-sectional survey was conducted in November 2010 with randomly selected households in Shivalaya and Harirumpur, both subdistricts of Manikganj district, Bangladesh. Arsenic contamination rates in these areas are around 50 per cent, making well-switching a simple and no-cost mitigation option. Wellswitching refers to switching from using an arsenic-contaminated tube well (usually painted red) to sharing the arsenic-safe tube well (usually painted green) of a neighbouring household.

Participants and procedures. Criteria for study participation were as follows: (a) being at risk of drinking arsenic-contaminated water (i.e. having an arsenic-contaminated tube well or, for non-owners of tube wells, collecting or having previously collected water from a contaminated well) and (b) having access to a neighbouring arsenic-safe tube well.

We first determined the broader areas of the surveys by selecting three unions (one sub-district has approximately 10 unions): Arua, Ulail and Balla. The unions had to be geographically separated 
from one another to avoid information contamination in the controlled trial later. We randomly selected a total of 12 villages within the study areas (villages with $<30$ households were excluded) that were separable into four regions, allowing for four different intervention conditions for the later trial. Sample size estimations with GPOWER (Erdfelder et al., 1996) yielded a total sample size of 280 households to detect a medium effect at the Type I error probability of 0.05 and a power of 0.95 for the four intervention arms. Based on a prior study of well-switching (Inauen et al., 2013), we also adjusted for the exclusion of users of arsenic-safe water options in the later trial. We thus aimed at interviewing 95 households in each of the four areas.

A team of 10 professional Bangladeshi interviewers was recruited to conduct the survey. The interviewers were extensively trained in a 5-day workshop to conduct structured face-to-face, 1-hour interviews. Particular importance was paid to the rehearsal of language in order to certify that each interviewer used the same vocabulary to explain the questions in an easily understandable manner for the rural participants. During the survey, a quality-control team answered any uncertainties the interviewers had and ensured the completeness of the questionnaires.

Households were randomly selected with the random-route method (Hoffmeyer-Zlotnik, 2003). For a given household, the interviewers first asked to speak to the person responsible for collecting drinking water (usually a woman). Then, interviewers assessed whether the household met the above-mentioned inclusion criteria. If yes, fully informed consent was obtained prior to conducting the interview. As is usually the case for survey research in rural low-income countries (e.g. Mosler et al., 2010), refusal was minimal, and only 8 persons $(2 \%)$ refused to participate. A total of 379 households were interviewed. The vast majority of participants were female $(355 ; 93.7 \%)$, and the average age was 37.3 years ( standard deviation $(S D)=11.8$ years). About half of the respondents were literate $(191 ; 50.4 \%)$ and had an average of 3.6 years of formal education $(S D=3.9$ years $)$. The mean monthly household income was 5228 Bangladeshi Taka (BDT; approximately US $\$ 63$; $S D=4211$ BDT).

Questionnaire. A structured questionnaire was developed and translated from English into Bengali. Re-translation into English revealed translation difficulties with some questions. These were resolved in round table discussions before and after the pretest. The questionnaire contained questions regarding water consumption, the behavioural determinants and sociodemographic characteristics.

Water consumption. Participants were asked how many vessels of which water source they collected for drinking on a typical day during the week preceding the survey. Because all respondents either collected all water from safe sources or all water from contaminated sources in the week prior to the survey, the final outcome was use (score $=1$ ) or non-use (score $=0)$ of arsenic-safe water for drinking.

Knowledge. This was assessed with a set of 18 'yes-no' questions. The questions were concerned with knowledge of which water sources contained arsenic, whether contaminated water was safe to drink, which medical conditions could be caused by arsenic and tasks for which it was okay to use arsenic-contaminated water. Furthermore, respondents were asked whether they knew the location of a safe water option in their village, whether it was safe to drink from a green-coloured tube well, whether arsenic can be removed by boiling and to name water sources that were free from arsenic. Each correct answer was assigned 1 point. In the end, all points were added to the score and transformed into the standardised value range of $0-1$.

All other psychological constructs were measured with several items each on Likert scales (5-point for unipolar items and 9-point for bipolar items), which were averaged. To ensure understanding of the questions, a hierarchical procedure was applied. Participants were first asked to choose one of three answer categories (e.g. rather like, rather dislike and rather neutral). Then, participants were offered more detailed 
response options in the category they had chosen (e.g. dislike very much, dislike and rather dislike). Example questions for each construct of the RANAS model are presented below. Note that all items were transformed into a value range of $0-1$ (or -1 to 1 for bipolar items), in order to facilitate interpretation of the unstandardised regression coefficients. A score of 0 (or -1 ) always represents the least favourable value for using arsenic-safe wells and 1 the most favourable. Therefore, for example, the perceived severity response options were as follows: $0=$ not at all severe, $0.25=$ not severe, $0.5=$ quite severe, $0.75=$ severe, $1=$ very severe.

Vulnerability. A total of 3 items were used to assess vulnerability. Participants were asked how high or low the chances were that they or someone in their family would develop arsenicosis, and how high their chances were of developing arsenicosis compared to persons of their sex and age $(-1=$ very low to $1=$ very high; Cronbach's alpha $=.84)$.

Severity. This was measured using 3 items. Participants were asked, 'Imagine that you contracted arsenicosis, how severely would it impact your life in general/your social life/your economic situation?' $(0=$ not at all severe to $1=$ very severe; Cronbach's alpha $=.87$ ).

Affective attitude. This construct was measured using 7 items. Participants were asked, for example, whether they liked collecting water from the safe well, whether they felt ashamed of collecting water from there or whether they liked the water taste $(-1=$ dislike very much to 1 = like very much; Cronbach's alpha $=.82$ ).

Instrumental attitude. Perceived expenditure of time and effort was measured using 2 items. For example, 'Do you think that collecting water from the arsenic-safe option is timeconsuming?' $(0=$ not at all time-consuming to $1=$ very time-consuming; Cronbach's alpha = .84). The scale was inverted so that low values reflected low attitudes (i.e. high effort/time) and high values reflected favourable attitudes (i.e. low effort/time).

Injunctive norm. This was assessed using 3 items. People were asked, for example,
'Overall, how much would people who are important to you approve or disapprove of you collecting water from the arsenic-safe water option?' (-1 = they (would) disapprove very much to 1 = they (would) approve very much; Cronbach's alpha $=.75)$.

Descriptive norm. A total of 2 items were used to assess this. Participants were asked to name the number of people outside their families or of their village who collected water from the safe water option $(0=$ almost nobody to 1 = almost everybody; Cronbach's alpha = .86).

Action self-efficacy. This was assessed using 3 items. Participants were asked, for example, how difficult or easy it was to find time to collect water from the arsenic-safe well ( -1 $=$ very difficult to 1 = very easy; Cronbach's alpha = .94).

Maintenance self-efficacy. Participants answered three questions related to how confident they felt about collecting water from the safe option 'even if they had to walk a long distance/the safe option was broken/they did not feel like collecting water' $(0=$ not at all confident to 1 = very confident; Cronbach's alpha $=.89$ ).

Recovery self-efficacy. A total of 3 items were used to assess people's recovery self-efficacy. People were asked, for example, 'Imagine that you stopped going to collect water from the safe well for several days. How confident are you to start collecting water from the safe option again?' $(0=$ not at all confident to $1=$ very confident; Cronbach's alpha $=.93$ ).

Action planning. This was measured with 3 items. Participants were asked, for example, 'Have you made a detailed plan when during the day to start collecting water from the arsenicsafe option?' $(0=$ no detailed plan at all to $1=$ very detailed plan; Cronbach's alpha $=.87$ ).

Coping planning. This was measured with 3 items. Participants were asked, for example, 'Have you made a detailed plan of what to do when the arsenic-safe option is broken?' $(0=$ no detailed plan at all to $1=$ very detailed plan; Cronbach's alpha $=.85$ ). 
Action control. A total of 6 questions assessed this. Participants were asked, for example, 'How much did you pay attention so you do not forget to collect water from the mitigation option?' $(0=$ no attention at all to $1=$ much attention; Cronbach's alpha $=.86$ ).

Forgetting. As a proxy to measure remembering, respondents were asked an open-ended question: 'How many times did you forget to collect water from the arsenic-safe option in the last week?'

Commitment. A total of 3 items were used to assess this. Participants were asked how important was it for them to collect water from the safe option, how committed they felt to collecting water from the safe well and how annoyed they felt if they forgot to collect water from there $(0=$ not at all to $1=$ very important/ committed/annoyed; Cronbach's alpha $=.76$ ).

Data analysis. All calculations were computed with IBM SPSS Statistics 20.0. Key behavioural factors were determined by logistic regressions of the use and non-use of arsenicsafe water for drinking. Outliers with residuals greater than two standard deviations were excluded from the model. In addition, cases with missing values were excluded.

\section{Results}

A total of $105(27.7 \%)$ participants reported using neighbouring arsenic-safe shallow tube wells for drinking. The intercorrelations of all psychological factors are presented in Table 1, and their frequencies are presented in Table 2. Note that action planning and action control had to be excluded from the analyses because they were only answerable for either non-users (action planning) or users (action control) of safe water options. Furthermore, forgetting was excluded because $354(93.4 \%)$ respondents reported zero forgetting.

The survey revealed medium knowledge about arsenic and arsenicosis. Despite this, perceived vulnerability regarding arsenicosis, on average, was rather low. Severity of the consequences of developing arsenicosis was consistently rated high. Participants, on average, reported rather low affective attitudes and high instrumental attitudes. Regarding social norms, participants stated medium strength beliefs that collecting water from neighbouring safe wells was expected by others (injunctive norm). On average, participants reported that some others were collecting water from arsenic-safe neighbouring wells (descriptive norm). The mean rating of the difficulty of the task of collecting arsenic-safe water was near zero (neither easy nor difficult). People reported rather high confidence in their ability to collect water from the arsenic-safe water options even if barriers arose (maintenance self-efficacy) or if they stopped collecting water from there for a while (recovery selfefficacy). Despite rather high self-efficacy, participants' coping plans were not clearly defined. Finally, people were only rather committed to collecting arsenic-safe water.

Intercorrelations between study variables were generally low (see Table 1) but indicated some interrelatedness between action self-efficacy and instrumental attitude $(r=.67, p<$ $.001)$ and between maintenance self-efficacy and recovery self-efficacy $(r=.70, p<.001)$. An analysis of the variance inflation factors (VIFs) in the regression model indicated acceptable multi-collinearity: All VIFs were below 2, except for the self-efficacy factors VIF $_{\text {max }}=$ 2.73 for recovery self-efficacy).

Results of the logistic regression revealed that the psychological factors predicted the use and non-use of neighbouring arsenic-safe shallow tube wells very well (Table 2 ). The strongest predictors of the use and non-use of neighbouring arsenic-safe shallow tube wells were the descriptive norm, commitment, vulnerability (unexpectedly negatively associated) and recovery self-efficacy. Furthermore, higher maintenance self-efficacy, instrumental attitudes and injunctive norms were in line with the use of safe tube wells, but, unexpectedly, lower affective attitudes were negatively related. 


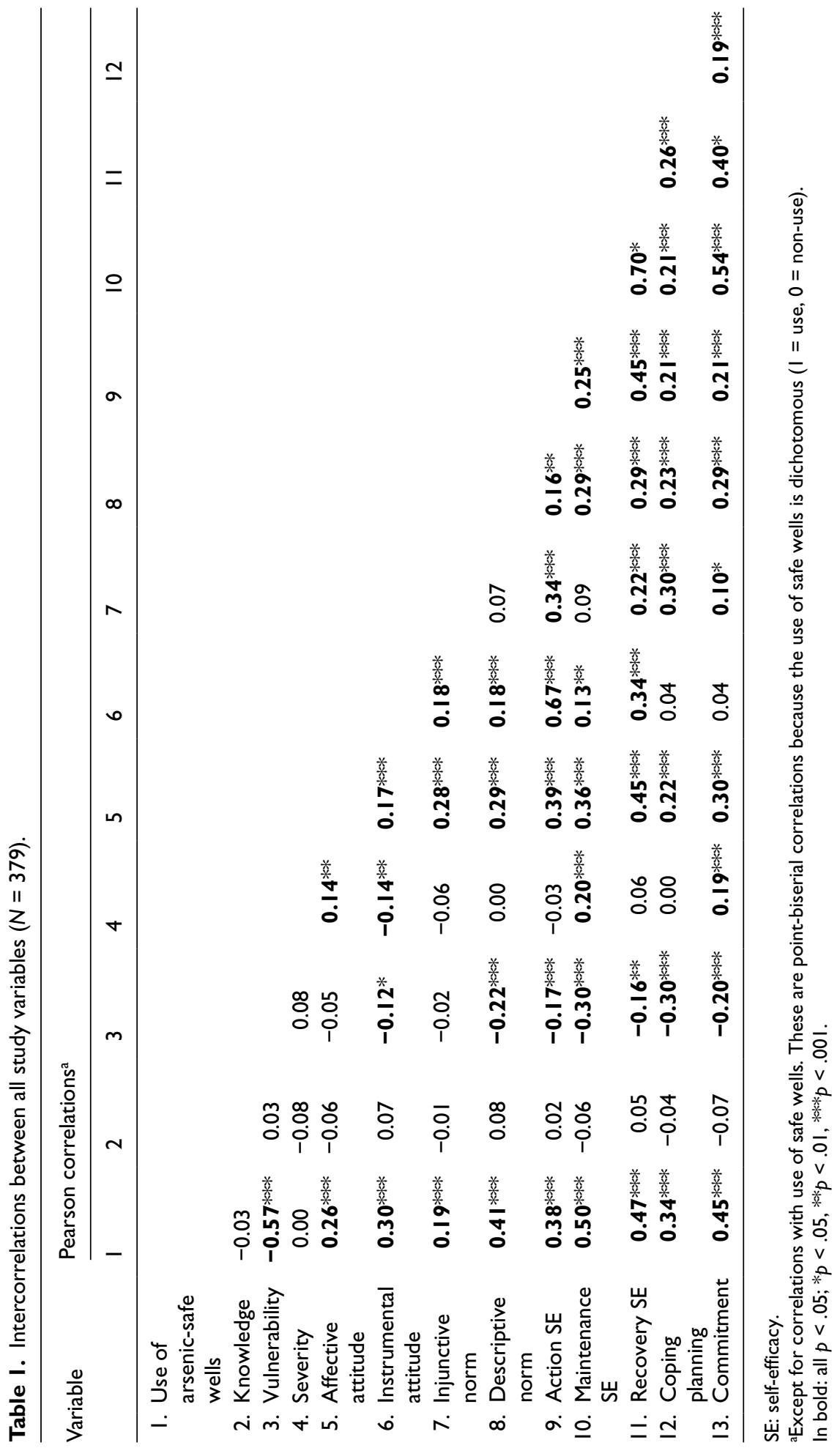


Table 2. Descriptive statistics and logistic regressions of the use of neighbouring arsenic-safe shallow tube wells on psychological factors $(n=363)$.

\begin{tabular}{|c|c|c|c|c|c|c|c|}
\hline \multirow[t]{2}{*}{ Factors } & \multirow[t]{2}{*}{ M } & \multirow[t]{2}{*}{$S D$} & \multirow[t]{2}{*}{ B } & \multirow[t]{2}{*}{ OR } & \multicolumn{2}{|c|}{$95 \% \mathrm{Cl}$ of $\mathrm{OR}$} & \multirow[t]{2}{*}{$p$} \\
\hline & & & & & LL & UL & \\
\hline Knowledge & 0.50 & 0.16 & 0.52 & 1.68 & 0.04 & 69.98 & .786 \\
\hline Vulnerability & -0.12 & 0.53 & -7.67 & 0.00 & 0.00 & 0.01 & .000 \\
\hline Severity & 0.82 & 0.13 & -2.56 & 0.08 & 0.00 & 16.33 & .349 \\
\hline Affective attitude & 0.33 & 0.31 & -3.10 & 0.05 & 0.00 & 0.49 & .011 \\
\hline Instrumental attitude & 0.53 & 0.29 & 3.73 & 41.70 & 1.19 & 1463.25 & .040 \\
\hline Injunctive norm & 0.47 & 0.36 & 2.79 & 16.22 & 1.54 & 170.78 & .020 \\
\hline Descriptive norm & 0.42 & 0.23 & 8.55 & $5 \mid 48.83$ & 86.37 & 306929.69 & .000 \\
\hline Action self-efficacy & 0.09 & 0.55 & -0.13 & 0.88 & 0.16 & 4.91 & .883 \\
\hline Maintenance self-efficacy & 0.45 & 0.25 & 4.60 & 99.89 & 1.06 & 9458.11 & .047 \\
\hline Recovery self-efficacy & 0.51 & 0.25 & 7.60 & 1996.73 & 14.64 & 272255.54 & .002 \\
\hline Coping planning & 0.22 & 0.18 & -2.54 & 0.08 & 0.00 & 3.17 & .178 \\
\hline $\begin{array}{l}\text { Commitment } \\
\text { Constant }\end{array}$ & 0.40 & 0.26 & $\begin{array}{r}8.17 \\
-18.30\end{array}$ & $\begin{array}{r}3518.69 \\
.000\end{array}$ & 73.32 & 168855.99 & $\begin{array}{l}.000 \\
.000\end{array}$ \\
\hline
\end{tabular}

SD: standard deviation; OR: odds ratio; Cl: confidence interval; LL: lower limit; UL: upper limit.

All variables ranged from 0 to $I$, except the bipolar variables (vulnerability, affective attitude, injunctive norm and action self-efficacy) that ranged from $-I$ to $I$. Use of arsenic-safe water option $=I$, non-use $=0$. Nagelkerke $R^{2}=.894$; correct classifications $95.3 \%$.

\section{Discussion}

The results of Part 1 of this research indicate that the use of neighbouring arsenic-safe shallow tube wells is much more likely for people with higher commitment, stronger descriptive norms and higher maintenance and recovery self-efficacies. Furthermore, vulnerability was strongly associated with using arsenic-safe wells. However, the direction of the relationship was unexpectedly negative. The most likely explanation of this finding is reverse causality - people who consume arsenic-safe water consequently feel less vulnerable to developing arsenicosis. Longitudinal research should investigate this more conclusively.

To determine the highest impact potential of a behaviour change intervention, we followed Mosler's (2012) suggestion to consider both the strength of association with the target behaviour and the means of the psychological factors in the population. The factors with highest behaviour change potentials with regard to the means $(M<0.50)$ were vulnerability, affective attitude, injunctive norm, descriptive norm, action self-efficacy, maintenance self-efficacy, coping planning and commitment (see Table 2). Combining the information regarding the means and the strength of association with using arsenic-safe wells, the following three factors emerged as having the highest impact potential in behaviour change intervention: commitment, the descriptive norm and recovery self-efficacy. In addition, the affective attitude may have an impact; however, the negative association of using arsenic-safe wells in the regression suggests complex interactions with other psychological factors. We, therefore, did not target this factor. Similarly, we did not target vulnerability due to the unclear causality of the relation of this factor with the use of arsenic-safe wells.

The RANAS model suggests a set of BCTs that can modify each of the determinants identified above (Mosler, 2012). See Figure 1 for details on programme, intervention and BCTs. To increase commitment, we selected prompts and implementation intentions. Prompts are simple reminders of a target behaviour (e.g. seatbelt use, Thyer and Geller, 1987) that are assumed to increase its accessibility and 


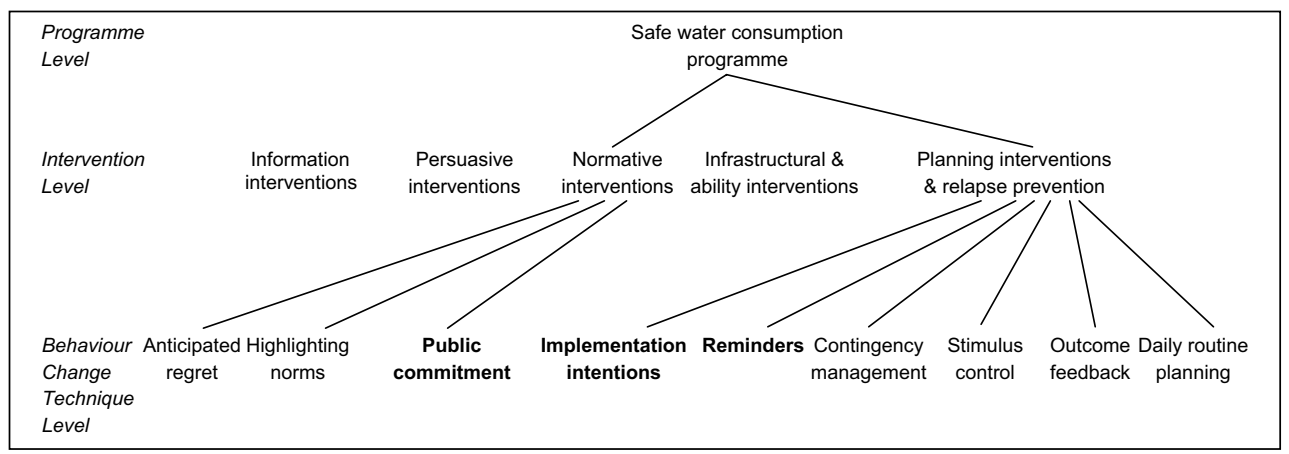

Figure I. Tree diagram showing programme, intervention and behaviour change technique levels of description (adapted from the RANAS model; Mosler, 20I2). The behaviour change techniques that were tested in addition to risk information in this study are displayed in bold.

RANAS: Risk, Attitudes, Norms, Abilities, Self-regulation.

strengthen commitment (Tobias, 2009). Implementation intentions are detailed plans that link situations with actions (Gollwitzer, 1999). BCTs are low-cost and have been shown to effectively change a series of health-related and other behaviours (e.g. Adriaanse et al., 2011; Cox et al., 2005; Tobias, 2009). To enhance descriptive and injunctive norms, the public commitment intervention was selected from the norm BCTs (Mosler, 2012). Group interventions are frequently and popularly applied in Bangladesh. Furthermore, public commitment not only highlights norms, but should increase personal commitment also. Finally, Mosler (2012) suggests that maintenance self-efficacy and recovery self-efficacy can be enhanced by coping planning. However, in order not to overload this intervention, these factors were targeted in a second intervention phase that will be published elsewhere. In the following, the efficacy of the developed interventions in comparison with an informationonly control will be tested.

\section{Part 2:Testing the effectiveness of theory-based and evidence- based interventions}

To test the assumption that the interventions, which were developed on the basis of theory and evidence, are more effective in promoting wellswitching than an informational intervention, we conducted a cluster-randomised controlled trial. It was hypothesised that information would render smaller behaviour change effects than information combined with reminders $(\mathrm{H} 1)$, information combined with reminders and implementation intentions (H2) and all interventions combined with public commitment (H3). Furthermore, it was assumed that more wellswitching will be observed in the condition that combined implementation intentions, reminders and information compared to reminders and information alone (H4). Finally, we hypothesised that the combination of all interventions with a public commitment will lead to a greater proportion of well-switching than information, reminders and implementation intentions (H5).

\section{Methods}

The cluster-randomised controlled trial was conducted from November 2010 to April 2011 in Shivalaya, Bangladesh. Four clusters of 2-4 villages were randomly assigned to an information-only control group or to one of three theory-based intervention groups: (a) information and reminders; (b) information, reminders and implementation intentions; (c) information, reminders, implementation intentions and public commitment. This trial was conducted and 
reported in compliance with the Consolidated Standards of Reporting Trials (CONSORT) procedures (Moher et al., 2001) and the guidelines for the publication of intervention studies (Marks, 2010).

Clusters and participants. In principle, participants of the first part of this study were the same as in the second part, and the assessment described in Part 1 formed the baseline of the trial. However, one additional inclusion criterion for the randomised controlled trial was non-use of arsenic-safe water at baseline. Consequently, 105 users were excluded. The remaining 274 participants roughly corresponded to the required sample size (see above). But to adjust for possible attrition, an additional four villages adjacent to the selected villages were included, and 96 additional randomly selected households were surveyed at the baseline. In total, 370 households in 16 villages were included in the trial (see Figure 2 for the participants flow). Most participants were female $(350 ; 94.6 \%)$ and were, on average, 36.6 years old ( $S D=12.2$ years). In all, 183 were literate $(49.5 \%)$ and had attended school for 3.6 years $(S D=4.0)$. The mean monthly household income was 6670 BDT (approximately US\$80; $S D=3610$ BDT).

Interventions. All interventions were delivered by health promoters of a local non-governmental organisation, Christian Commission for Development in Bangladesh (CCDB). Each of them received $4000 \mathrm{BDT}$ (approximately US\$50) for their services during this 1-month intervention. The 5 female promoters (18-25 years old) lived in the study areas and were trained by the first author (J.I.) and a local collaborator to correctly provide the interventions. These were delivered in February 2011. At each visit, promoters obtained fully informed consent. Thereafter, promoters conducted the intervention session, which lasted from 20 to 60 minutes depending on the intervention condition (each BCT required approximately 20 minutes). Participants in the public commitment condition were also invited to join the commitment session held in their village 2 weeks after the promoter's visit. A local supervisor was employed for quality control and to assist the promoters. The elements of the interventions are described below (intervention manuals can be found at http://www.eawag.ch/ forschung/siam/schwerpunkte/soziale_systeme/ Beh_Change_Guideline_2012.pdf). Note that all materials were designed so they could also be understood by illiterate participants.

Information on arsenic, arsenicosis and arsenicsafe drinking water options. This element of the intervention was the control condition and the basis of the three theory-based intervention arms. The promoters explained the following content to the participants by demonstrating a booklet with pictograms and photographs. First, promoters informed participants about arsenic in shallow tube well water. Second, it was explained that arsenic can have adverse health effects, and these effects were described. Finally, participants were told where arsenicsafe water can be found in their communities (green-marked shallow tube wells), and in general (all major arsenic-safe water sources available in Bangladesh).

Reminders. A set of two reminders was developed - a poster and a tag. The poster was designed to remind participants in the key situation (just before their drinking water was finished) to collect their water from the safe option. It depicted an almost empty kalosh (local vessel for water collection (pl. kolshi)) and a woman who goes to collect water from a green-marked tube well instead of a red-marked one, which was crossed out.

The tag was developed to remind participants not to collect drinking water from the red-marked tube well. It contained pictograms showing the purposes for which the water should not be used: drinking directly, boiling and then drinking or cooking. Furthermore, the tag displayed the purposes for which the water can be used (e.g. bathing, washing dishes).

Promoters first explained the contents of the poster and then installed it at the place within 


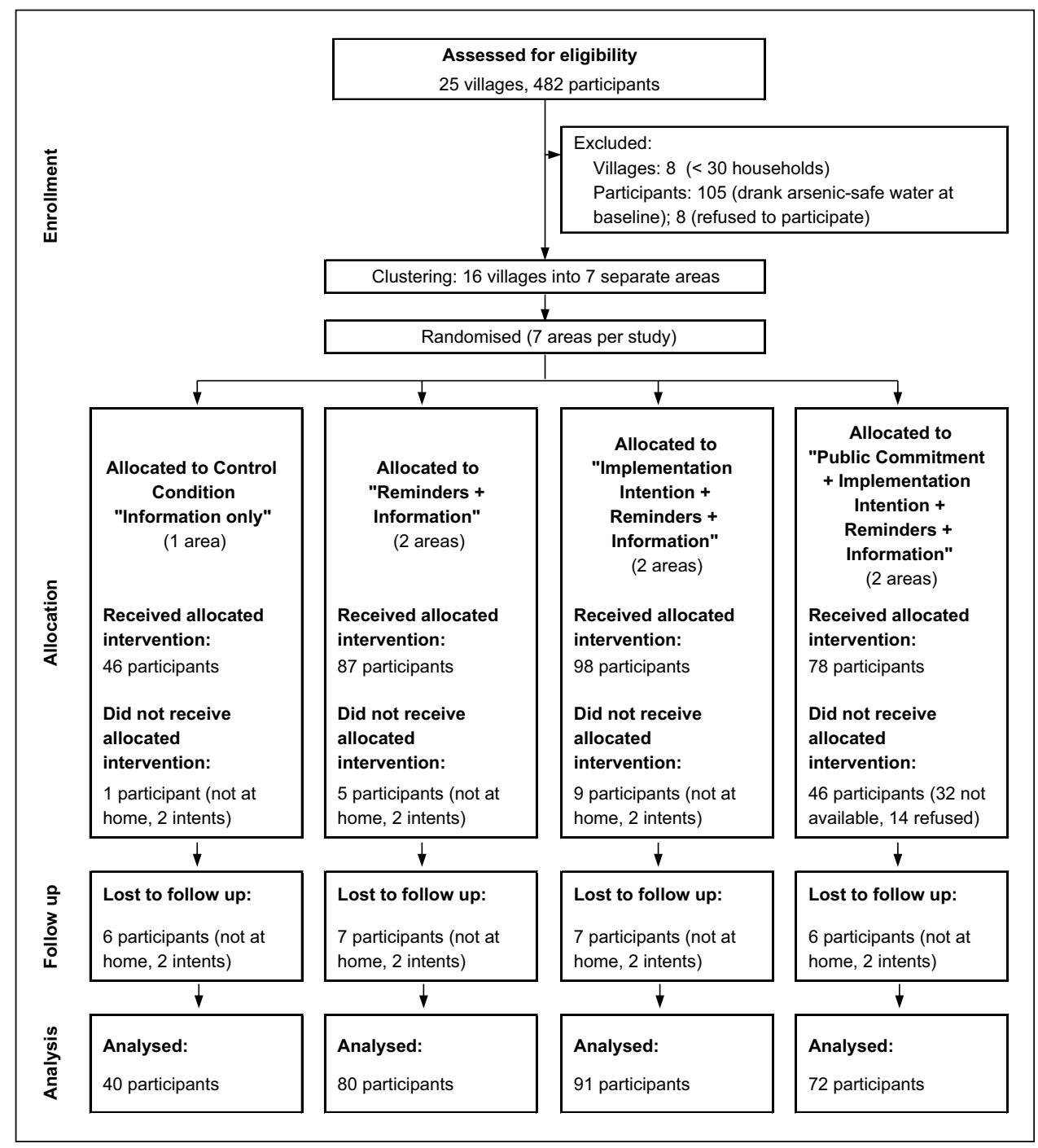

Figure 2. Participant flow through the cluster-randomised controlled trial.

the household where participants kept their kolshi. Thereafter, promoters explained the contents of the tag and installed it at the arseniccontaminated tube wells, which the participants reported to use.

Implementation intention. Implementation intentions are specific plans for where, when and how to perform a behaviour (Gollwitzer and Brandstätter, 1997). It is a special challenge to conduct these interventions for people with low literacy who are not used to living by the clock. We used pictograms with typical tasks during the day for the 'when' part of the plans (e.g. sunrise, breakfast and bathing). First, promoters asked participants how many times a day they would have to collect water at their neighbour's arsenic-safe tube well. Then, participants were asked to specify a situation before or after which it would be best for them to collect water (e.g. before preparing lunch). Then, participants named a specific neighbour's green-marked tube well from where they 
committed to collecting their drinking water. Subsequently, they specified how many kolshi they would collect each time they went and for which purpose (drinking, cooking or both). In the end, participants were asked to repeat the plan out loud after the promoter, to sign the implementation intention form by thumbprint and to keep it safe.

Public commitment. Participants in this condition were invited by the promoter to join the public commitment session that was held in their respective villages approximately 1 week after the promoter visit. The sessions were part informational and part commitment. First, using posters, a team of two promoters and the supervisor again explained the information about arsenic, arsenicosis and arsenic-safe drinking water to the group of participants. Thereafter, participants were asked to commit themselves to only drink arsenic-safe water from now on. The participants who committed were asked to read their implementation intentions to the group. At the end of the 2-hour session, tea and biscuits were offered.

Data collection. Data during the follow-up in April 2011 were collected by face-to-face interviews as described in Part 1. Special care was taken to interview the same participants as were interviewed during the baseline. Again, the final outcome was the $1=$ use or $0=$ non-use of arsenic-safe water for drinking.

Data analysis. First, a randomisation check was performed by comparing intervention and control groups on all psychological factors at baseline and sociodemographic variables by analyses of variance (ANOVAs) or Chi-square tests (for dichotomous measures). For the former, the Welch statistic was used due to inhomogeneous variances. Omega square $\left(\omega^{2}\right)$ was computed as a measure of effect size. To test for potential biases due to attrition, dropouts were compared in order to study households on all baseline measures with independent samples t-tests and Chi-square tests. In the main analyses, logistic regressions of the use or non-use of arsenic-safe drinking water were conducted. Intervention dummy variables (each intervention vs control group) served as independent variables. Variables with significant baseline differences between intervention groups were entered as covariates.

\section{Results}

Randomisation check. No baseline differences between intervention groups were found for knowledge, severity, instrumental attitude, action self-efficacy, recovery self-efficacy, commitment, income, education or literacy. However, significant effects were found for vulnerability $(F(3,125.549)=16.13, p<.000$, $\left.\omega^{2}=.13\right)$, affective attitude $(F(3,128.258)=$ $\left.8.53, p<.000, \omega^{2}=.08\right)$, injunctive norm $(F(3$, $\left.130.776)=4.96, p=.003, \omega^{2}=.04\right)$, descriptive $\operatorname{norm}\left(F(3,126.438)=7.01, p<.000, \omega^{2}=.05\right)$, maintenance self-efficacy $(F(3,126.226)=$ 5.391, $\left.p=.002, \omega^{2}=.04\right)$, coping planning $\left(F(3,131.566)=4.03, p=.009, \omega^{2}=.03\right)$, age $\left(F(3,129.523)=3.24, p=.024, \omega^{2}=.02\right)$ and distance to an arsenic-safe well $(F(3,129.116)$ $\left.=3.43, p=.019, \omega^{2}=.02\right)$. Although most effects were small, these variables were entered as covariates in the main analysis.

Attrition check. In total, 61 households did not receive the allocated interventions, and 26 households were out of reach during the followup (see Figure 2). No differences between dropouts and included households were found for knowledge, vulnerability, severity, instrumental attitude, the self-efficacies, coping planning, age, income, education or literacy. However, dropouts displayed significantly higher affective attitudes $(t(180)=-3.67, p<.000, r=$ $0.17)$, higher injunctive norms $(t(182)=-2.21$, $p=.028, r=0.16)$, significantly lower descriptive norms $(t(171)=2.35, p=.020, r=0.18)$ and lower commitment $(t(149)=2.04, p=.044$, $r=0.16)$. All effects were small. Finally, the number of dropouts differed significantly between the intervention and control groups $\left(\chi^{2}\right.$ $=35.31, d f=3, p<.000)$. In the public 
commitment condition, the dropout rate was particularly high $(52 ; 60 \%)$, but it was small in the other groups (implementation intention: 18\%; reminders: $14 \%$; control: $8 \%$ ).

Main analysis. In the information-only control condition, 7 participants (18\%) had switched to an arsenic-safe neighbouring shallow tube well at follow-up. In the intervention group with information and reminders, 35 (44\%) had switched. A total of 60 households $(66 \%)$ with information, reminders and implementation intentions had switched. Unexpectedly, only 13 households (18\%) who had received all the interventions plus public commitment had switched to safe water. Logistic regressions of the use of arsenic-safe drinking water at followup supported H1 and H2. Compared to information only, participants with additional reminders were 3.10 times more likely to switch to an arsenic-safe water source $(B=1.13$, standard error $(S E)=0.50, d f=1, p=.024)$, whereas participants with an additional implementation intention were 8.05 times more likely to switch $(B=$ 2.09, $S E=0.56, d f=1, p<.000)$. The additional implementation intention tended to increase the likelihood of well-switching compared to reminders and information alone $(\operatorname{Exp}(B)=$ 2.09; $B=0.74, S E=0.38, d f=1, p=.052)$. However, contradicting H3 and H5, participants with all interventions and a public commitment were not more likely to switch to an arsenic-safe well than participants in the control condition $(\operatorname{Exp}(B)=1.48 ; B=0.39, S E=0.79, d f=1, p=$ $.620)$ and were less likely to switch than households in the implementation intention condition $(\operatorname{Exp}(B)=.136 ; B=-2.00, S E=0.42, d f=1, p$ $<.000)$. None of the covariates achieved significance in any of the analyses.

\section{Discussion}

In a cluster-randomised controlled trial, theorybased and evidence-based interventions were compared to an information-only control condition regarding their effectiveness in promoting the use of arsenic-safe wells. In line with our hypotheses, more people switched to arsenicsafe wells when theory-based and evidencebased interventions were delivered in addition to risk information. In particular, implementation intentions when combined with information and reminders proved effective in promoting well-switching. An additional public commitment intervention, however, was not more effective than information alone. This result is surprising because it seems that the commitment session had a detrimental effect on the effects of the other interventions. Possible reasons for this may be that social influence processes may have taken place during the sessions, for example, opinion leaders who were opposed to collecting from neighbouring safe wells may have been present in the sessions. In addition, perhaps the commitment session was conducted too soon after the promoter's visit and may have caused reactance. Furthermore, in line with this assumption is the fact that nonattendance of the public commitment sessions was high, indicating that only the higher motivated participants attended these sessions. Making their already formed commitments public may have caused further reactance.

\section{Overall discussion}

We argued that developing behaviour change interventions based on psychological theory and evidence will enhance the effects of a risk information intervention to encourage switching to arsenic-safe wells. In the first part, this research revealed that commitment, the descriptive norm, self-efficacy and perceived vulnerability are the main predictors of the probability of using neighbouring arsenic-safe wells. Interventions developed to target these factors increased behaviour change effects of a risk information intervention by up to 48 per cent. The most successful interventions to promote well-switching were implementation intentions in combination with information and reminders delivered by local health promoters. Public commitment had an unexpected negative effect on people switching to arsenic-safe wells. 
Group-based interventions should, therefore, be applied with caution until further studies identify when, for whom and why group interventions may be beneficial or detrimental.

Regarding the factors that were associated with using arsenic-safe wells, the results of this study highlight the importance of descriptive norms and self-efficacy. This is in line with previous research on safe water consumption that demonstrated the importance of the perception that safe water collection is typical in the community and that people are confident of being able to collect safe water in the long term (Huber et al., 2011; Mosler et al., 2010). Furthermore, commitment was highly predictive of arsenic-safe water use. This finding supports the assumption that this is an important factor to consider in this context and also perhaps for other behaviours (Tobias, 2009). This notion is further underlined by the high behaviour change impact of interventions that targeted commitment strengthening.

Overall, the medium to strong increase in the behaviour change effect of the theory-based and evidence-based interventions support the usefulness of an applied approach of intervention development. This strengthens the assumption that theory is useful for intervention development (Michie and Johnston, 2012). It shows that the psychological factors that need to be modified in the intervention can be identified by considering whether theoretically proposed factors are associated with the target behaviour and how they are distributed in the population in need of change. Without evidence from a baseline assessment, any other factor from the theoretical model could have been targeted in the intervention, for example, severity. However, according to this approach, such an intervention is likely to have produced little effect in this case because most people in the population already rated arsenicosis as severe. Furthermore, severity was not significantly associated with using arsenic-safe wells.

Of the applied interventions, implementation intentions in combination with information and reminders produced the strongest behaviour change effects. This is in line with the vast literature that documents the strong effects of implementation intentions on changing a series of behaviours (e.g. Adriaanse et al., 2011; Cox et al., 2005; Tobias, 2009). Particularly encouraging, with regard to behaviour change in developing countries, is the fact that these low-cost implementation intentions were also effective in a format tailored to illiterate persons. Regarding the question of cost-effectiveness of interventions, it would be beneficial to investigate whether the behaviour change effects are altered if implementation intentions are applied without the reminders and risk information. More important, future research should focus on the mechanisms of change, that is, to investigate whether the interventions indeed changed behaviour by modifying the proposed psychological factors. Finally, it is important to identify possible moderators of these intervention effects. For example, the HAPA suggests that post-motivational interventions only enhance health behaviour change for people with prior intentions (Schwarzer, 2008). In turn, weaker intervention effects on well-switching are expected for persons who need to walk greater distances to safe wells (Opar et al., 2007).

The small sample size of the control group may be considered a limitation of this study. Despite this, most intervention effects still reached significance because they were sufficiently large. It is possible, however, that differences in well-switching rates between the public commitment group and the control group could have been detected in a larger sample. In any case, the differences between these two groups are very small.

The fact that the interventions were developed based on a cross-sectional investigation of factors associated with the use of arsenic-safe wells may be seen as a further limitation of this study. Certainly, causality of the relationship between the psychological factors and behaviour cannot be ascertained by these designs. It may, therefore, be fruitful to apply a longitudinal design for assessing the psychological factors and predicting the target behaviour. However, 
the additional time and resources required for such a longitudinal intervention development period must be considered. Future studies should compare the cost-effectiveness of interventions developed from cross-sectional data and panel data.

\section{Funding}

This research was funded by Eawag: Swiss Federal Institute of Aquatic Science \& Technology, Switzerland.

\section{References}

Abraham C and Michie S (2008) A taxonomy of behavior change techniques used in interventions. Health Psychology 27: 379-387.

Adriaanse MA, Vinkers CD, De Ridder DT, et al. (2011) Do implementation intentions help to eat a healthy diet? A systematic review and metaanalysis of the empirical evidence. Appetite 56(1): 183-193.

Argos M, Kalra T, Rathouz PJ, et al. (2010) Arsenic exposure from drinking water, and all-cause and chronic-disease mortalities in Bangladesh (HEALS): A prospective cohort study. Lancet 376(9737): 252-258.

Bandura A (1997) Self-Efficacy: The Exercise of Control. New York: Freeman.

Brewer NT, Chapman GB, Gibbons FX, et al. (2007) Meta-analysis of the relationship between risk perception and health behavior: The example of vaccination. Health Psychology 26(2): 136-145.

Cialdini RB (2003) Crafting normative messages to protect the environment. Current Directions in Psychological Science 12: 105-109.

Claassen L, Henneman L, Kindt I, et al. (2010) Perceived risk and representations of cardiovascular disease and preventive behaviour in people diagnosed with familial hypercholesterolemia: A cross-sectional questionnaire study. Journal of Health Psychology 15: 33-43.

Conner M and Sparks P (1996) The theory of planned behavior and health behaviors. In: Conner $\mathrm{M}$ and Norman P (eds) Predicting Health Behaviour: Research and Practice with Social Cognition Models. Buckingham: Open University Press, pp. 121-162.

Conner M, Rhodes RE, Morris B, et al. (2011) Changing exercise through targeting affective or cognitive attitudes. Psychology \& Health 26(2): 133-149.

Cox CD, Cox BS and Cox DJ (2005) Long-term benefits of prompts to use safety belts among drivers exiting senior communities. Journal of Applied Behavior Analysis 38(4): 533-536.

Erdfelder E, Faul F and Buchner A (1996) GPOWER: A general power analysis program. Behavior Research Methods Instruments \& Computers 28: $1-11$.

Gollwitzer PM (1999) Implementation intentions: Strong effects of simple plans. American Psychologist 54(7): 493-503.

Gollwitzer PM and Brandstätter V (1997) Implementation intentions and effective goal pursuit. Journal of Personality and Social Psychology 73(1): 186-199.

Graf J, Meierhofer R, Wegelin M, et al. (2008) Water disinfection and hygiene behaviour in an urban slum in Kenya: Impact on childhood diarrhoea and influence of beliefs. International Journal of Environmental Health Research 18: 335-355.

Heri S and Mosler H-J (2008) Factors affecting the diffusion of solar water disinfection: A field study in Bolivia. Health Education \& Behavior 35(4): 541-560.

Hoffmeyer-Zlotnik JHP (2003) New sampling designs and the quality of data. Advances in Methodology and Statistics/Metodološki Zvezki 19: 205-217.

Huber AC and Mosler H-J (2012) Determining behavioral factors for interventions to increase safe water consumption: A cross-sectional field study in rural Ethiopia. International Journal of Environmental Health Research 23(2): 96-107.

Huber AC, Bhend S and Mosler H-J (2012) Determinants of exclusive consumption of fluoridefree water: A cross-sectional household study in rural Ethiopia. Journal of Public Health 20(3): 269-278.

Inauen J, Hossain MM, Johnston RB, et al. (2013) Acceptance and use of eight arsenic-safe drinking water options in Bangladesh. PLoS One 8(1): e53640.

Johnston RB and Sarker MH (2007) Arsenic mitigation in Bangladesh: National screening data and case studies in three upazilas. Journal of Environmental Science and Health: Part A 42(12): 1889-1896.

Kraemer SM and Mosler H-J (2010) Persuasion factors influencing the decision to use sustainable 
household water treatment. International Journal of Environmental Health Research 20: 61-79.

Lawton R, Conner M and Parker D (2007) Beyond cognition: Predicting health risk behaviors from instrumental and affective beliefs. Health Psychology 26(3): 259-267.

Marks DF (2009) Editorial: How should psychology interventions be reported? Journal of Health Psychology 14: 475-489.

Marks DF (2010) Publication guidelines for intervention studies in the Journal of Health Psychology 15: 5-7.

Michie S and Johnston M (2012) Theories and techniques of behaviour change: Developing a cumulative science of behaviour change. Health Psychology Review 6(1): 1-6.

Michie S, Johnston M, Francis J, et al. (2008) From theory to intervention: Mapping theoretically derived behavioural determinants to behaviour change techniques. Applied Psychology 57(4): 660-680.

Michie S, Richardson M, Johnston M, et al. (2013) The Behavior Change Technique Taxonomy (v1) of 93 hierarchically clustered techniques: Building an international consensus for the reporting of behavior change interventions. Annals of Behavioral Medicine 1-15. doi: 10.1007/s12160-013-9486-6

Moher D, Schulz KF and Altman DG; the CONSORT Group (2001) The CONSORT statement: Revised recommendations for improving the quality of reports of parallel-group randomized trials. Annals of Internal Medicine 134: 657-662.

Mosler H-J (2012) A systematic approach to behavior change interventions for the water and sanitation sector in developing countries: A conceptual model, a review, and a guideline. International Journal of Environmental Health Research 22: 431-449.

Mosler H-J, Blöchliger OR and Inauen J (2010) Personal, social, and situational factors influencing the consumption of drinking water from arsenicsafe deep tubewells in Bangladesh. Journal of Environmental Management 91(6): 1316-1323.

Opar A, Pfaff A, Seddique AA, et al. (2007) Responses of 6500 households to arsenic mitigation in Araihazar, Bangladesh. Health \& Place 13: 164-172.
Radtke T, Scholz U, Keller R, et al. (2011) Smoking is ok as long as I eat healthily: Compensatory health beliefs and their role for intentions and smoking within the Health Action Process Approach. Psychology \& Health 27: 91-107.

Schultz PW, Nolan JM, Cialdini RB, et al. (2007) The constructive, destructive, and reconstructive power of social norms. Psychological Science 18(5): 429-434.

Schwarzer R (2008) Modeling health behavior change: How to predict and modify the adoption and maintenance of health behaviors. Applied Psychology: An International Review 57(1): 1-29.

Schwarzer R and Luszczynska A (2008) How to overcome health-compromising behaviors. European Psychologist 13(2): 141-151.

Sheeran P and Orbell S (1999) Augmenting the theory of planned behavior: Roles for anticipated regret and descriptive norms. Journal of Applied Social Psychology 29(10): 2107-2142.

Smith AH and Steinmaus CM (2009) Health effects of arsenic and chromium in drinking water: Recent human findings. Annual Review of Public Health 30: 107-122.

Sniehotta FF, Scholz U and Schwarzer R (2005) Bridging the intention-behaviour gap: Planning, self-efficacy, and action control in the adoption and maintenance of physical exercise. Psychology \& Health 20: 143-160.

Thyer BA and Geller ES (1987) The 'buckle-up' dashboard sticker: An effective environmental intervention for safety belt promotion. Environment and Behavior 19: 484-494.

Tobias R (2009) Changing behavior by memory aids: A social psychological model of prospective memory and habit development tested with dynamic field data. Psychological Review 116(2): 408-438.

Tobias R and Berg M (2011) Sustainable use of arsenic-removing sand filters in Vietnam: Psychological and social factors. Environmental Science \& Technology 45(8): 3260-3267.

Van Geen A, Ahsan H, Horneman AH, et al. (2002) Promotion of well-switching to mitigate the current arsenic crisis in Bangladesh. Bulletin of the World Health Organization 80(9): 732-737. 